\title{
Ophthalmochirurgie der letzten 3 Dekaden - ein persönlicher Rückblick
}

\author{
Gerhard F. Kieselbach
}

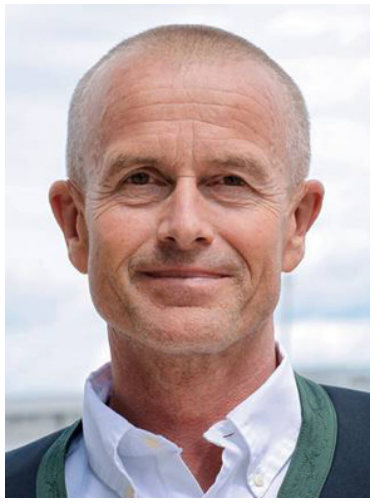

Mittlerweile - wir schreiben das Jahr 2017 - ist die Vitrektomie weder ein Sakrileg noch ein angezweifeltes Verfahren. Als ich vor über 30 Jahren meine ersten Vitrektomien durchführte, war das Entfernen des Glaskörpers nicht nur aufgrund des Instrumentariums ein Abenteuer. Es gab keine schweren Perfluorcarbone, ein Endolaser zur intraoperativen Verwendung war in Innsbruck noch nicht in Verwendung und das intraoperative Kaltlicht war ähnlich dem Fernlicht meines R4 (Abb. 1, Renault mit 5 Türen), also vertraut, aber auch nicht erhellend.

Die Qualität der Guillotine-Messer - verglichen mit den heutigen - im "Stripper“ war auch bei neuen schon eher zögerlich und verbesserte sich auch nicht

Anmerkung: Um Missverständnissen vorzubeugen, stelle ich fest, dass im Text zwar der Chirurg verwendet wird, aber damit sind alle Chirurginnen mit einbezogen, da es gerade in Österreich eine große Zahl an hervorragenden Netzhautchirurginnen gibt.

\section{Univ.-Prof. Dr. G. F. Kieselbach ( $₫)$}

Universitätsklinik für Augenheilkunde, Medizinische

Universität Innsbruck, Anichstraße 35, 6020 Innsbruck, Österreich

Gerhard.kieselbach@i-med.ac.at nach regelmäßigen Sterilisationen. „Disposable“ war damals noch ein echtes Fremdwort. Meine ersten Vitrektomien wurden mit dem originalen Klöti-Stripper durchgeführt, ein Ungetüm, welches Aspiration, Irrigation und Licht in einem vereinte und einen aufgenähten Trokar brauchte, der mit einer Sicherheitsnaht versehen war, um am Ende der Operation schnell verschlossen werden zu können (Abb. 2, KlötiStripper mit Infusion und Kaltlichaufsatz im Original, Oertli).

Andererseits wurde schon damals mit Trokaren gearbeitet, was ein Vierteljahrhundert später als Innovation gefeiert wurde. Ein eklatanter persönlicher Sprung war kurz darauf die Umstellung auf minimal drei Scleraöffnungen und indirekte Beobachtung mit dem ersten BIOM von Spitznas (Abb. 3).

Es gab keine Diskussion, ob primäre Vitrektomie oder Plombenchirurgie. Primär wurde - basierend auf den Publikationen von Kreissig und den Vorträgen von Lund, zu denen wir Innsbrucker Augenärzte der Klinik ohne Zwang regelmäßig gesandt wurden - eine Plombe mit Kryokoagulation durchgeführt.

Anschließend (eher gezwungenermaßen) oder initial bei mehreren Defekten der Netzhaut war eine Umgürtung des Auges mit diversen Materialien, Größen und Formen Standard. Erst beim Auftreten einer PVR, die so selten nicht war - da ja ausreichend und ausgiebig die Kryosonden eingesetzt wurde -, hat man eine Vitrektomie überlegt und ohne Perfluocarbon, aber mit Titannägel zur vorübergehenden Fixation der eingerollten Netzhaut in Form eines Hochamtes zelebriert. Auch deswegen, weil der Patient in Narkose am OP-Tisch angeschnallt und auf den Kopf gestellt wurde um die subretinale Flüssigkeit durch eine Luftblase zu drainieren.

Eine Art von Pfingsten erlebte ich 1986 bei meinem ersten von mehreren Besuchen in Rotterdam und Antwerpen bei Dr. R. Zivojnovic, der nicht nur das Buch 


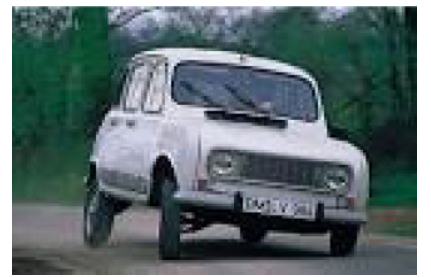

Abb. 1 Renault 4

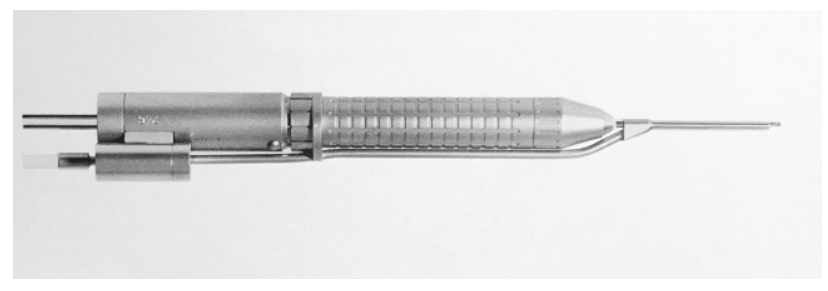

Abb. 2 Klöti Stripper, Oertli 19

„Silicone Oil in Vitreoretinal Surgery“ schrieb, sondern mir unter vielen anderen zwei wesentliche Prinzipien mitgab:

Fülle niemals Silikonöl ein, bevor die Netzhaut nicht anliegt und alles Blut oder anderes Material aus dem Auge entfernt ist! Beherzigt man diesen Leitspruch, steigt die Erfolgsrate enorm.

Sein zweiter Leitspruch, den ich noch immer versuche, allen Kollegen mitzugeben, lautet: Der Chirurg muss alle Geräte und Instrumente kennen und beherrschen, denn wenn es nicht klappt, ist immer der Chirurg schuld.

Zeitgleich war 1985 die Expression des Linsenkern mit Implantation einer im Sulcus fixierten Hinterkammerlinse schon Standard, die 12-15 Nylonnähte 10.0 erlaubten es jedoch nicht, Patienten nach nur einer Woche zu entlassen. Auch die kurz darauf eingeführte Phakoemulsifikation war noch lange Zeit nicht ohne Nähte vorstellbar.

Ein Visus über 0,5 disqualifizierte damals die Patienten von jeder Kataraktoperation, da nur harte Linsenkerne problemlos exprimierbar waren. Die Diskussion, ob eine intrakapsuläre oder extrakapsuläre Kataraktextraktion (ECCE) durchgeführt werden sollte, war noch nicht beendet und nur der Vorteil, den Glaskörper zu schonen, gab der ECCE den entscheidenden Vorteil, um sich in fast ganz Europa zu etablieren.

Die Vorstellung, den Glaskörper anzutasten, ja zu entfernen, war damals in den Augen der etablierten Chirurgen fast unvorstellbar und wurde bis in die 90er angezweifelt, ja von wenigen vehement verdammt.

Glaskörperblutungen bei Diabetikern wurden noch mit Binocolus und stationärer Schonung über 4 bis 12 (!) Wochen behandelt.

Emotionsgeladene Diskussionen bei Fachtagungen zu all diesen Punkten waren in meiner Erinnerung der Normalfall.

Erwähnenswert ist die Tatsache, dass in der 80ern noch immer nicht alle Augenchirurgen Handschuhe trugen, um besser mit den 10.0 Nylonfäden arbeiten zu können und alle Abdeckungen aus Baumwolle waren, unvorstellbar heute auch, dass die Irrigations-/ Aspirationsleitungen nicht bei jedem Patienten ausgetauscht wurden.

Die phantastische Weiterentwicklung der intraokularen Chirurgie war aber in den darauffolgenden Jahren beispielhaft für die gesamte Chirurgie. Besonders die Einführung der Perfluorcarbone durch Stanley Chang 1988 (Intraoperative perfluorocarbon liquids in the management of proliferative vitreoretinopathy) und deren langsame Verbreitung auch in Europa gab der Vitrektomie einen massiven Schub.

Rückblickend waren unsere heroischen Operationen, wie die Extraktion von chorioretinalen Neovaskularisationen bei feuchter Makuladegeneration, zwar anatomisch wunderschöne Operationen, funktionell überzeugten diese Operationen aber schon damals nur in ausgewählten Fällen. Ähnlich waren die Versuche der Makulatranslokation, entweder nach Machemer mit $360^{\circ}$ Retinotomie oder aber in limitierter Form mit Sclerafältelung. Weitere Versuche mit hoher Außenwirkung und geringem Vorteil für die betroffenen Patienten waren die interne Pigmenttranslokation, also Pigmentblätter von der Peripherie unter die Makula verlagern und die RON, die radiäre Optikoneurotomie, ein Versuch bei Zentralvenenverschlüssen eine neue kollaterale Verbindung zu erschaffen.

Der große Vorteil all dieser Operationen bestand meines Erachtens vor allem darin, dass wir Operateure ein immer besseres Verständnis der intraokulären Strukturen erlernten. Viele Patienten mit intraokulären Fremdkörpern oder anderen perforierenden Verletzungen haben davon profitiert. Waren in den 80ern perforierende Verletzungen durch Autounfälle noch unser täglich Brot, die Versorgung solcher Augen wesentlicher Bestandteil unserer 7 bis 10 Nachtdienste pro Monat, so verringerte sich diese Zahl durch die Gurtenpflicht und deren Akzeptanz in den folgenden Jahren dramatisch.

Eine andere sehr wesentliche Erkenntnis resultierte aus der Makulalochchirurgie und Entfernung epiretinaler Membranen sowie der Membrana limitans interna (MLI). Die nächste große Diskussion entfachte sich daraufhin Ende der 90er, inwieweit Luft und GAS zu Gesichtsfelddefekten führt. Dies spitzte sich dann zu, als Indocyaningrün (ICG) zum Anfärben der MLI erfolgreich eingesetzt wurde und nur wenig später in zahlreichen Arbeiten die Toxizität von ICG hervorgehoben wurde.

Schade an diesen Diskussionen war der Umstand, dass echte Konzentrationen an der Netzhaut niemals gemessen werden konnten und damit bis heute nicht sicher feststeht, ab welcher Menge und Dauer diese zweifelsfrei hilfreiche Substanz zu Schäden führt.

Parallel zu den intrakolären Entwicklungen der Chirurgie ging die Anwendung der verschiedensten Laserwellenlängen in der Behandlung aller möglichen Netzhauterkrankungen. 
Abb. 3 Biom heute und vor 25 Jahren
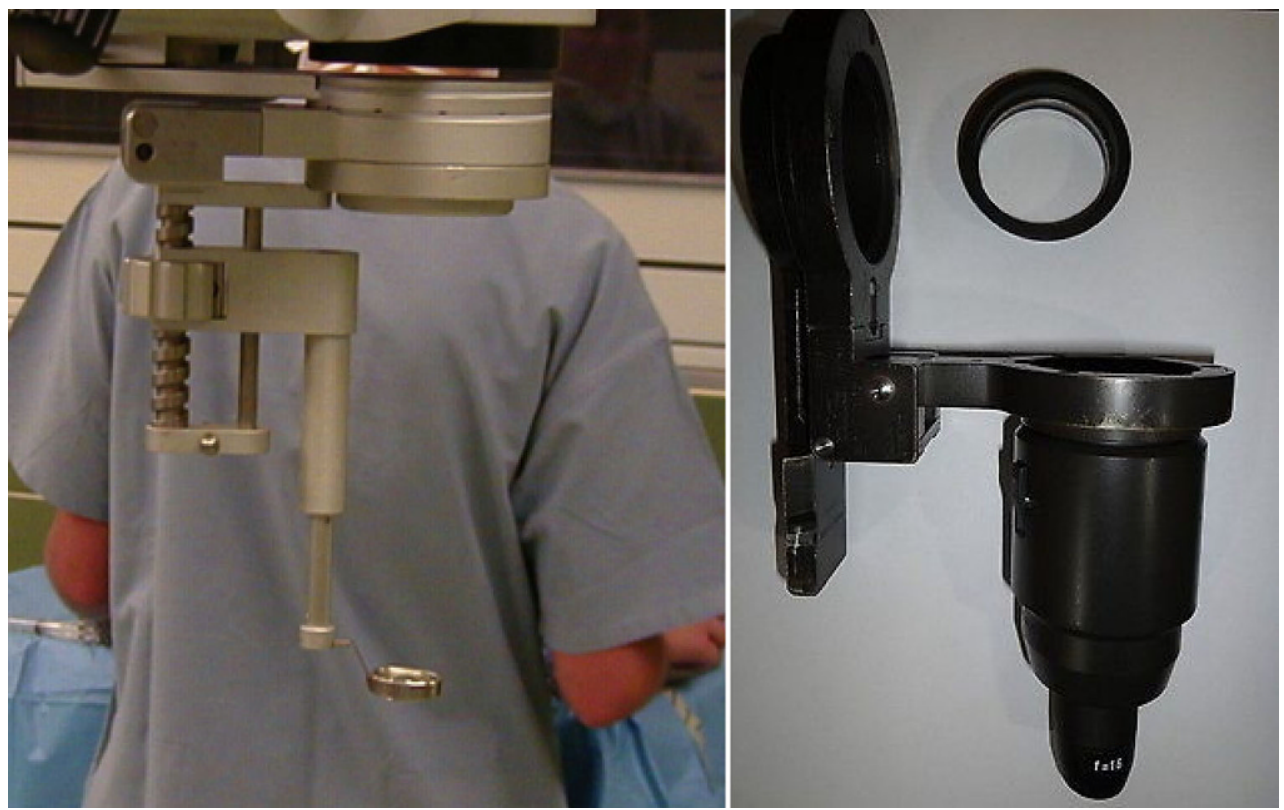

Mit den Publikationen der ETDRS (1985) war über Dekaden der Laser in allen möglichen Farben, Größen und Intensitäten die einzige Behandlungsoption für diabetische Komplikationen am Auge.

Auch rückblickend ist man noch immer betroffen, wie vielen Patienten man trotz zahlreicher anstrengender Lasersitzungen bei Venenverschlüssen nicht helfen konnte und wie viele Patienten mit proliferativer Retinopathie heute mit einem Gesichtsfeld wie Retinopthia - pigmentosa - Patienten auskommen müssen.

Eine Diskussion der 90er war, ob man die Choroidale Neovaskularistion, sofern klassisch bei einem Visus von 0,4 , mit dem Laser behandeln soll, damit der Visus von 0,1 dann stabil bleibt. Die Macular Photocoagulation Study zeigte, dass die Laserbehandlung der klassischen CNV nach 2 Jahren im Vergleich zu unbehandelten Augen besser war, allerdings mit einer Rezidivrate von über $54 \%$. Logischerweise gab es für beide Seiten Befürworter, dieser Disput konnte jedoch 1998 durch die Einführung der photodynamischen Therapie beendet werden.

Neben der Laserbehandlung der CNV, der prophylaktischen Laserung von Drusen, der Röntgenbestrahlung der AMD war auch die Dialyse von Patienten mit Makuladegeneration eine weitere Sackgasse.

Der Gedanke, der mich 2000 beim Anblick des ersten Humphrey OCT beschäftigte, war: Was tue ich mit all diesen vielen Informationen? Aus heutiger Sicht ein klägliches Ansinnen, da ich mir eine vollständige Untersuchung ohne OCT nicht mehr vorstellen kann.

Die Ära der aVEGF-Medikamente hat ein wesentliches netzhautchirurgisches Kapitel beendet, was zwar für viele Patienten sehr hilfreich ist, aber dennoch auch die Weiterentwicklung chirurgischer Verfahren deutlich gebremst hat.

Nach dem heutigen Stand der Dinge ist auch die Implantation von subretinalen oder epiretinalen lichtempfindlichen Platinen noch kein Standardeingriff geworden, da die technischen Fragen die Antworten noch immer mehr als exponential übertreffen.

Es ist mehr als faszinierend, in dieser Epoche grandioser Entwicklungen - aber auch vieler chirurgischer Sackgassen - arbeiten zu können und dann die Möglichkeiten zu haben, diese in Studien zu testen. Auch wenn viele klinische Studien nicht die erhofften Ergebnisse zeitigen, so ist doch jeder Versuch, seine Abläufe zu optimieren, ein wichtiger Schritt in die richtige Richtung und kann trotzdem sehr befriedigend verlaufen.

Neues zu entwickeln und anzuwenden zum Wohle des Patienten ist wohl das Schönste, das einem in seinem Beruf passieren kann.

Es zeigt aber auch, dass nur die stetige Adaption des eigenen Handelns die einzige Konstante sein kann.

Interessenkonflikt G.F. Kieselbach gibt an, dass kein Interessenkonflikt besteht. 